\title{
PERIODICITY AND INDECOMPOSABILITY
}

\author{
W. T. INGRAM
}

(Communicated by James E. West)

\begin{abstract}
In this paper we characterize the existence of periodic points of odd period greater than one for unimodal mappings of an interval onto itself. The interesting juxtaposition of this condition with the occurrence in inverse limits of the well-known Brouwer-Janiszewski-Knaster continuum is explored. Also obtained is a characterization of indecomposability of certain inverse limits using a single unimodal bonding map.
\end{abstract}

\section{INTRODUCTION}

This paper grew out of investigations of inverse limits on $[0,1]$ using logistic bonding maps and inverse limits on $[0,1]$ using families of piecewise linear bonding maps. In each case, a "standard" indecomposable continuum, the socalled Brouwer-Janiszewski-Knaster continuum (throughout this paper we will refer to this continuum as the B-J-K continuum) [8, p. 204], kept occurring. It showed up often enough that the author isolated a theorem (Theorem 2) which produces the phenomenon. Then, the author observed that, by perturbing the condition which gives rise to the B-J-K continuum, for a certain class of mappings (which includes the logistic family, unimodal maps, and the tent family) a characterization of the existence of periodic points of odd period for this class of mappings is obtained (Theorem 6). By considering composites and looking at appropriate subintervals on which the restricted mapping belongs to the appropriate class, this theorem yields the existence of periodic points of periods which are odd multiples of powers of two. Dynamicists have long been interested in periodic points whose period is not a power of two (see [10], for example). The author's interest arises from the connection with the existence of indecomposable continua in the inverse limit [1], [6].

By a continuum we mean a compact, connected subset of a metric space. By a mapping we mean a continuous function. If $X_{1}, X_{2}, X_{3}, \cdots$ is a sequence of metric spaces and $f_{1}, f_{2}, f_{3}, \cdots$ is a sequence of mappings (called bonding maps) such that, for each positive integer $i, f_{i}: X_{i+1} \rightarrow X_{i}$ then by the inverse limit of the inverse limit sequence $\left\{X_{i}, f_{i}\right\}$ is meant the subset of the

Received by the editors June 8, 1993; presented to the American Mathematical Society in the Special Session on Continuum Theory and Dynamical Systems, San Antonio, Texas, January, 1993.

1991 Mathematics Subject Classification. Primary 54H20, 54F15, 58F03, 58F08.

Key words and phrases. Periodic point, indecomposable continuum, unimodal mapping, inverse limit. 
product space, $\prod_{i>0} X_{i}$, to which the point $\left(x_{1}, x_{2}, x_{3}, \cdots\right)$ belongs if and only if $f_{i}\left(x_{i+1}\right)=x_{i}$. The inverse limit of the inverse limit sequence $\left\{X_{i}, f_{i}\right\}$ is denoted $\lim _{\longleftarrow}\left\{X_{i}, f_{i}\right\}$. Here, the product space is metrizable with the metric $d(x, y)=\sum_{i>0} \frac{d_{i}\left(x_{i}, y_{i}\right)}{2^{i}}$ where, for each $i, d_{i}$ is a metric for $X_{i}$ bounded by one. It is well known that, when the spaces $X_{i}$ are continua and the bonding mappings are continuous, the inverse limit exists and is a continuum. In case, for each $i, X_{i}=X$ and $f_{i}=f$, we denote the inverse limit by $\lim _{\{}\{X, f\}$. In case $Y$ is a subcontinuum of $X$ and $f[Y]$ is a subset of $Y$, for convenience, we sometimes denote $\stackrel{\lim }{\longleftarrow}\{Y, f \mid Y\}$ simply by $\underset{\lim }{\longleftarrow}\{Y, f\}$. A point $x$ is said to be a periodic point for a mapping $f$ provided there is a positive integer $n$ such that $f^{n}(x)=x$. If $n$ is the least positive integer $k$ such that $f^{k}(x)=x$ then we say that $x$ is periodic of period $n$. A mapping of a continuum is monotone provided each point inverse is a continuum. A mapping of an interval $[a, b]$ onto itself is called unimodal on $[a, b]$ provided $f$ is not monotone and there is a point $c$ of $(a, b)$ such that $f(c)$ belongs to the set $\{a, b\}$ and $f$ is monotone on $[a, c]$ and $[c, b]$. (This is a slightly more general definition than that of a unimodal map [3, p. 63].)

In this paper we will concern ourselves with unimodal mappings of intervals onto themselves. There are four cases to consider when studying unimodal mappings of an interval $[a, b]$ onto itself: (1) $f(b)=a$, (2) $f(a)=a$,

Type (1)

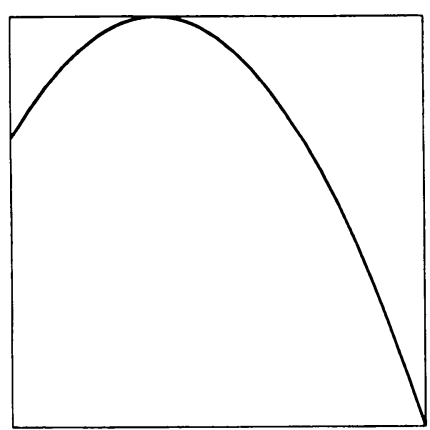

Type (3)

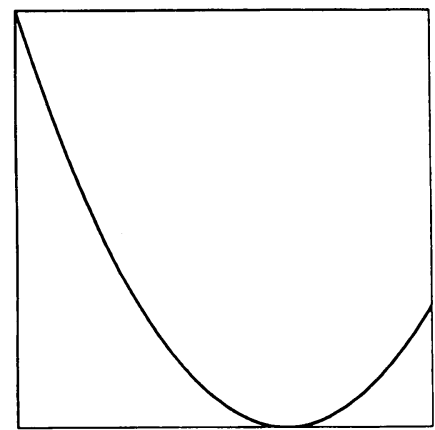

Type (2)

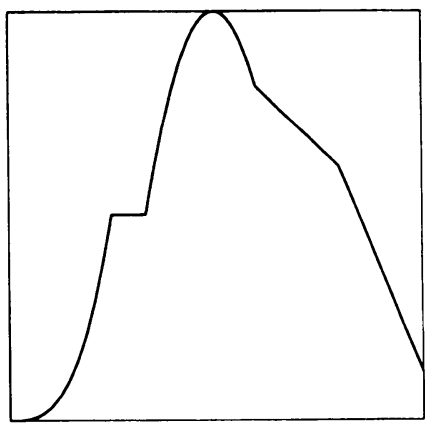

Type (4)

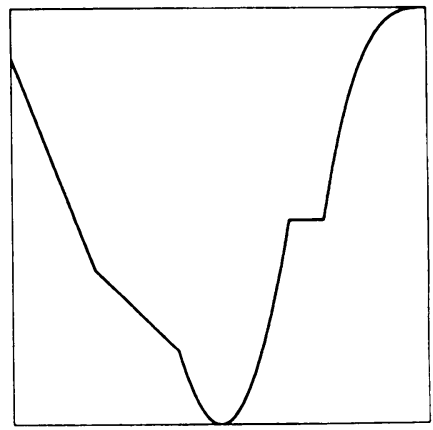

FigURE 1 
(3) $f(a)=b$, and (4) $f(b)=b$. These we will reference by Type (1), Type (2), Type (3), and Type (4). These four types are illustrated by four "typical" pictures shown in Figure 1. It is evident that each unimodal mapping of Type (3) is topologically conjugate to one of Type (1) and each one of Type (4) is topologically conjugate to one of Type (2). Since we are mainly interested in inverse limits along with dynamics and since topologically conjugate mappings yield the same dynamic behavior and homeomorphic inverse limits, we will limit our study to those of Type (1) and Type (2). For mappings of these two types, denote by $c$ a point between $a$ and $b$ such that $f(c)=b$ and by $p$ the fixed point of $f$ in $[c, b]$. If $f$ is a Type (2) unimodal mapping and $f(b) \leq c$ then $[f(b), b]$ is thrown onto itself by $f$ and $\lim _{\longleftarrow}\{[f(b), b], f \mid[f(b), b]\}$ is sometimes called the core of $\lim _{\longleftarrow}\{[a, b], f\}$.

\section{BenNetT's TheOREM}

In a beautiful Master's Thesis at the University of Tennessee, Ralph Bennett [2] proved a number of basic theorems about inverse limits on intervals. In particular, he proved a theorem which we only slightly generalize here as Theorem 1. This theorem gives the overall structure of the inverse limit and shows that the understanding of inverse limits using Type (2) unimodal bonding maps amounts to understanding the structure of the core because the core is the inverse limit on $[f(b), b]$ using the restriction of $f$ to $[f(b), b]$ which is a Type (1) unimodal map provided $f(b)<c$.

Theorem 1 (Bennett). Suppose $f$ is a mapping of the interval $[a, b]$ onto itself and $d$ is a number between $a$ and $b$ such that (1) $f[d, b]$ is a subset of $[d, b],(2) f \mid[a, d]$ is monotone, and (3) there is a positive integer $j$ such that $f^{j}[a, d]=[a, b]$. Then $\lim _{\longleftarrow}\{[a, b], f\}$ is the union of a topological ray $R$ and a continuum $K$ such that $\bar{R}-R=K$.

Proof. Let $M=\lim _{\longleftarrow}\{[a, b], f\}$ and $K=\lim \{[d, b], f\}$. For each postive integer $n$, let $\alpha_{n}$ denote the inverse limit using the restriction of $f$ on subintervals of $[a, b]$ determined by (1) the $n$th factor space is the interval $[a, d] ;(2)$ if $i$ is less than $n$, the $i$ th factor space is $f^{n-i}[a, d]$; and (3) if $i$ is greater than $n$, the $i$ th factor space is the $(i-n)$ th preimage of $[a, d]$ under the monotone mapping $f \mid[a, d]$. Let $R=\alpha_{1} \cup \alpha_{2} \cup \alpha_{3} \cup \cdots$. Note that, for each positive integer $j, \alpha_{j}$ is a subset of $\alpha_{j+1}$ and each $\alpha_{j}$ is an arc, so $R$ is a ray. To see that each point of $K$ is a limit point of $R$, note that if $x$ and $y$ are points of $M$ with $x_{n}=y_{n}$ then $d(x, y)<2^{-n}$. Then, if $n$ is a positive integer and $x$ is in $K, x_{n}$ is in $[d, b]$. By condition (3) of the hypothesis of this theorem, there are a point $y$ of $[a, d]$ and a positive integer $j$ such that $f^{j}(y)=x_{n}$. There is a point $p$ of $\alpha_{n+j}$ whose $(n+j)$ th coordinate is $y$, so $d(x, p)<2^{-n}$. Thus, $K$ is a subset of $\bar{R}$. On the other hand, if $x$ is a point of $M$ not in $R$ then $x$ is not in $\alpha_{n}$ for any $n$. Consequently, $x_{n} \geq d$ for each $n$, so $x$ is in $K$. Thus, $K=\bar{R}-R$.

\section{B-J-K CONTINUA}

In this section we demonstrate the existence of the B-J-K continuum in certain inverse limits. For a picture indicating this continuum, see, e.g., [8, p. 204]. 
This continuum is homeomorphic to an inverse limit on the interval $[0,1]$ using the mapping $f$ defined by $f(x)=4 x(1-x)$ as the only bonding map. More generally, James F. Davis has shown that if $g$ is a confluent mapping of $[0,1]$ of degree 2, then $\lim \{I, g\}$ is homeomorphic to $\lim \{I, f\}$, [4].

Theorem 1 applies to a class of mappings which includes those unimodal mappings of Type (2) with the additional properties that $f(b)<c$ and, for some $j>1, f^{j}(b)>c$. In Theorems 4 and 5 in this section, the hypotheses require that $f(b)<c$ and $f^{2}(b)>c$. Theorems 2 and 3 below address the nature of the core of certain inverse limits with unimodal bonding mappings of Type (2). In these inverse limits with Type (2) mappings, the core is an inverse limit with unimodal bonding mappings of Type (1). Recall that $p$ denotes the fixed point for $f$ in $(c, b]$.

Theorem 2. Suppose $f$ is a Type (1) unimodal mapping of an interval $[a, b]$ onto itself and $q$ is a point of $(c, p]$ such that $f^{2}(q)=q$ and $f(a)=q$. Then the inverse limit of the inverse limit system $\{[a, b], f\}$ is the union of two B-J-K continua intersecting at a point or an arc.

Proof. Note that, under the hypothesis of Theorem 2, the intervals $[a, f(q)]$ and $[q, b]$ are each mapped onto the other by $f$. Thus $f^{2} \mid[a, f(q)]$ and $f^{2} \mid[q, b]$ are both degree 2 confluent maps throwing the respective intervals onto themselves. Thus by Davis's theorem [4], $\underset{\lim }{\longleftarrow}\left\{[a, f(q)], f^{2}\right\}$ and $\lim _{\longleftarrow}\left\{[q, b], f^{2}\right\}$ are both homeomorphic to the B-J-K continuum. Each of these continua is homeomorphic to a subcontinuum of $M=\lim _{f[a, b], f\}}$ and their union is $M$. Their intersection is the $\operatorname{arc} \underset{\lim }{\longleftarrow}\{[q, \overleftarrow{f(q)}], f\}$ or is the point $(p, p, p, \cdots)$ in case $q=p$.

Theorem 3. Suppose $f$ is a Type (1) unimodal mapping of an interval $[a, b]$ onto itself and $q$ is a periodic point of period two in $(c, p)$ such that $f(a)=$ $f(q)$. Then the inverse limit of the inverse limit system $\{[a, b], f\}$ is the union of an arc $\alpha$ and two mutually exclusive B-J-K continua each intersecting $\alpha$ at one of its end points.

Proof. Under the hypotheses of this theorem, the intervals $[a, q]$ and $[f(q), b]$ are mutually exclusive and swapped by $f$ and, as in the proof of Theorem 2, the B-J-K continua are produced by this phenomenon. The arc $\alpha$ is

$$
\stackrel{\lim }{\longleftarrow}\{[q, f(q)], f\} \text {. }
$$

Theorem 4. Suppose $f$ is a Type (2) unimodal mapping of an interval $[a, b]$ onto itself and $q$ is a point of $(c, p]$ such that $f^{2}(q)=q$ and $f^{2}(b)=q$. Then the inverse limit of the inverse system $\{[a, b], f\}$ is the union of a topological ray $R$ and a decomposable continuum $C$ such that (1) $C$ is the union of two $B$-J-K continua intersecting at a point or an interval and (2) $\bar{R}-R=C$.

Proof. The ray $R$ can be obtained by applying Theorem 1. The continuum $C$ can be obtained by observing that $f \mid[f(b), b]$ satisfies the conditions of Theorem 2.

Theorem 5. Suppose $f$ is a Type (2) unimodal mapping of an interval $[a, b]$ onto itself and $q$ is a periodic point of period two in $(c, p)$ such that $f^{2}(b)=$ $f(q)$. Then the inverse limit of the inverse system $\{[a, b], f\}$ is the union of 
a topological ray $R$ and a decomposable continuum $C$ such that (1) $C$ is the union of an arc $\alpha$ and two mutually exclusive B-J-K continua each intersecting $\alpha$ at one of its end points and (2) $\bar{R}-R=C$.

Proof. The proof is similar to that of Theorem 4 except that one applies Theorem 3 instead of Theorem 2.

\section{PERIODIC POINTS OF ODD PERIOD}

In Theorem 6 we see that by perturbing the condition in Theorem 2 that $f(a)$ fall on a fixed point or a periodic point of period 2 we obtain, for the class of unimodal mappings, a characterization of the property of having a periodic point of odd period greater than one.

Lemma. Suppose $f$ is a Type (1) or Type (2) unimodal mapping of an interval $[a, b]$ onto itself, $q$ is the first fixed point for $f^{2}$ in $[c, b]$, and $f^{2}(b)<q$. Then there is a positive integer $k$ such that $f^{2 k+1}(c) \leq c$.

Proof. Since $f(c)=b$, if $f^{2}(b) \leq c$ then $f^{3}(c) \leq c$. Suppose $f^{2}(b)>c$. Note that, on the interval $[c, q]$, the function $f^{2}$ is non-decreasing and, except for the point $(q, q)$, its graph lies below the identity. Thus, $V=\left\{f^{2 n}(b) \mid n\right.$ is an integer and $\left.f^{2 n}(b)>c\right\}$ is a finite set, for if not, then the sequence $f^{2}(b), f^{4}(b), f^{6}(b), \cdots$ is a decreasing sequence bounded below, so it converges to a fixed point for $f^{2}$ in $[c, q)$. This contradicts the fact that $q$ is the first fixed point for $f^{2}$ in the interval $[c, p]$. Let $k$ be the least integer $n$ such that $f^{2 n}(b) \leq c$. Then, $f^{2 k+1}(c) \leq c$.

Theorem 6. Suppose $f$ is either a Type (1) or a Type (2) unimodal mapping of an interval $[a, b]$ onto itself and $q$ is the first fixed point for $f^{2}$ in $[c, b]$. Then, $f$ has a periodic point of odd period greater than 1 if and only if $f^{2}(b)<q$.

Proof. Suppose $f$ is a unimodal mapping of $[a, b]$ onto itself and $f^{2}(b)<q$. If $f$ is of Type (1) but not of Type (2), then $f$ has no fixed point in $[a, c]$. For convenience, we assume that if $f$ is of Type (2) then $f$ has no fixed point in $(a, c]$ (the alternative would be to use the last fixed point for $f$ in $[a, c]$ in the place of $a$ in the argument which follows). If $f$ is of Type (2), again assuming that $f$ has no fixed point in $(a, c)$, it is easily seen that, for each positive integer $n>1$, there is a point $y$ between $a$ and $c$ such that $f^{n}(y)=b>y$. If $f$ is of Type (1) and the point $a$ is not a periodic point of odd period for $f$, then, for each $k, f^{2 k+1}(a)>a$. Consequently, whether $f$ is of Type (1) or of Type (2), either the point $a$ is a periodic point of odd period for $f$ or, for each positive integer $k$, there is a point $y$ of $[a, c)$ such that $f^{2 k+1}(y)>y$. Now, by the Lemma, whether $f$ is of Type (1) or of Type (2), there is a positive integer $k$ such that $f^{2 k+1}(c) \leq c$. Since $f^{2 k+1}(c) \leq c$ and for the same $k$ there is a point $y$ between $a$ and $c$ such that $f^{2 k+1}(y)>y, f^{2 k+1}$ has a fixed point $x$ in $[y, c]$. Since $f$ has no fixed point in $(a, c], x$ is a periodic point for $f$ of odd period greater than 1 .

On the other hand, suppose $f$ is of Type (1) and $f^{2}(b) \geq q$, i.e., $f(a) \geq q$. Then if $x$ is a point of the interval $[a, f(q)]$ then $f(x)$ is in $[q, b]$, while if $x$ is in $[q, b]$ then $f(x)$ is in $[a, f(q)]$. As a consequence, $f^{2}$ throws each of the intervals $[a, f(q)]$ and $[q, b]$ onto itself. Thus, if $x$ is in $[a, f(q)]$ and is a periodic point for $f$ of odd period, then $x$ is in $[q, f(q)]$. Similarly, 
if $x$ is in $[q, b]$ and is a periodic point for $f$ of odd period, then $x$ is in [ $q, f(q)]$. But, $f \mid[q, f(q)]$ is a monotone mapping which has no periodic point of odd period greater than one. So, unimodal mappings of Type (1) for which $f^{2}(b) \geq q$ have no periodic points of odd period greater than one. In case $f$ is of Type (2) and $f^{2}(b) \geq q$, if $x$ is a point of the segment $(a, f(b))$, there is a positive integer $n$ such that $f^{n}(x)$ belongs to $[f(b), b]$. Since $[f(b), b]$ is thrown into itself by $f$, the only points of $(a, f(b))$ fixed by any iterate of $f$ are fixed by $f$. On the interval $[f(b), b]$, the mapping $f$ is a unimodal mapping of Type (1) for which $f^{2}(b) \geq q$ and the previous case applies to produce the result that $f$ has no periodic points of odd period greater than one.

\section{INDECOMPOSABILITY}

In this section we investigate the occurrence of indecomposable continua in inverse limits of intervals using unimodal bonding maps. Indecomposable continua are known to be present in an inverse limit on intervals with a single bonding map when the bonding map has a periodic point whose period is not a power of 2 [7]. In general, indecomposability of a subcontinuum can be shown by finding an interval on which the conditions of Kuykendall's theorem [9, Theorem 2, p. 267] are satisfied. Kuykendall proved that $\lim _{\longleftarrow}\left\{X_{i}, f_{i}\right\}$ is indecomposable if and only if for each positive integer $i$ and each positive number $\epsilon$ there exist a positive integer $n>i$ and three points of $X_{n}$ such that if $L$ is a subcontinuum of $X_{n}$ containing two of them then

$$
d_{i}\left(x, f_{i} \circ f_{i+1} \circ \cdots \circ f_{n-1}(L)\right)<\epsilon
$$

for each $x$ in $X_{i}$. In most of the applications of this theorem in this paper, we find two subintervals, each of which is thrown onto the whole interval either by $f$ or by some composite of $f$. Here we characterize when a Type (1) unimodal mapping produces an indecomposable inverse limit and when a Type (2) unimodal mapping produces an indecomposable core.

Theorem 7. Suppose $f$ is a Type (1) unimodal mapping of an interval $[a, b]$ onto itself and $q$ is the first fixed point for $f^{2}$ in $[c, b]$. Then, $\lim _{\longleftarrow}\{[a, b], f\}$ is indecomposable if and only if $f(a)<q$.

Proof. Observe that if $f(a)<q$ then $f[a, c]$ contains $[p, b]$ where $p$ is the fixed point for $f$ in $[c, b]$, so $f^{2}[a, c]$ contains $[a, p]$. Hence, for each $k$, the point $b$ is a member of $f^{2 k+1}([a, c])$. By the lemma, there is a positive integer $k \geq 1$ such that $f^{2 k+1}(c)<c$. Since $f$ is of Type $(1), f^{2}(c)=a$, and, consequently, $f^{2 k-1}(a)<c$. Thus, $f^{2 k}([a, c])$ contains $[a, b]$. Since $f^{n}[c, b]$ contains $[a, b]$ for each $n, \lim _{\longleftarrow}\left\{[a, b], f^{2 k}\right\}$ is indecomposable. Thus, $\lim _{\longleftarrow}\{[a, b], f\}$ is indecomposable.

On the other hand, if $f(a) \geq q$, as observed in the proof of Theorem 6, $f^{2}$ throws each of the intervals $[a, f(q)]$ and $[q, b]$ onto itself. So, the inverse limit of the inverse system $\left\{[a, b], f^{2}\right\}$ is the union of $\lim _{\longleftarrow}\left\{[a, f(q)], f^{2}\right\}$ and $\underset{\lim }{\ln }\left\{[q, b], f^{2}\right\}$.

Theorem 8. Suppose $f$ is a Type (2) unimodal mapping of an interval $[a, b]$ onto itself, $f$ has no fixed point between $a$ and $c$, and $q$ is the first fixed point 
for $f^{2}$ in $[c, b]$. Then, the core of $\lim _{\longleftarrow}\{[a, b], f\}$ is indecomposable if and only if $f^{2}(b)<q$.

Proof. Because $f$ has no fixed point between $a$ and $c$, it is easy to see that the interval $[f(b), b]$ is mapped onto itself by $f$. The core of $\lim \{[a, b], f\}$ is $\lim \{[f(b), b], f\}$. On the interval $[f(b), b], f$ is a unimodal mapping of Type (1) which satisfies the hypothesis of Theorem 7, and this theorem follows.

Remark. In Theorem 8, we assume that $f$ has no fixed point between $a$ and $c$. Unimodal mappings of Type (2) present minor technical difficulties if they have fixed points other than $a$ in $[a, c]$ and $f(b)$ falls on or between fixed points in $(a, c]$ (see, e.g., the Type (2) mapping of Figure 1). If this is the case, then the sequence $f(b), f^{2}(b), f^{3}(b), \cdots$ converges to a fixed point $a^{\prime}$ of $f$. If $f(b) \leq a^{\prime}$, then $\lim \{[f(b), b], f\}$ is indecomposable. To see this, consider the three points $f(b), c$, and $b$. It is easy to see that $f$ throws both $[c, b]$ and $[f(b), b]$ onto $[f(b), b]$. However, $f$ may not throw $[f(b), c]$ onto $f[(b), b]$, but $\left[a^{\prime}, b\right]$ and thus $[c, b]$ is a subset of $f[f(b), c]$, so $f^{2}$ throws $[f(b), c]$ onto $[f(b), b]$. By Kuykendall's theorem, the inverse limit is indecomposable. On the other hand, if $f(b)>a^{\prime}$, then $\lim _{\longleftarrow}\left\{\left[a^{\prime}, b\right], f\right\}$ is indecomposable. For the three points $f(b), c$, and $b$, if $x$ and $y$ are two of them then $\operatorname{cl}\left(\bigcup_{j>0} f^{j}[x, y]\right)=\left[a^{\prime}, b\right]$. Again, by Kuykendall's theorem, the inverse limit is indecomposable. Note that, if $a^{\prime}>a$, then $\lim _{\longleftarrow}\left\{\left[a, a^{\prime}\right], f\right\}$ is an arc which intersects but does not lie in this indecomposable continuum.

\section{FULl FAMILIES}

This section depends on results from Devaney [5] and Collet and Eckmann [3]. The reader who is not already familiar with the theory of kneading sequences and full families [5] (transition families [3]) is referred to these two sources for information. Such families are interesting dynamically and include such families of mappings of $[0,1]$ as $S_{\lambda}(x)=\lambda \sin (\pi x)$ for $0 \leq \lambda \leq 1$ and $s_{\lambda}(x)=\sin (\lambda \pi x)$ for $0 \leq \lambda \leq 1$ not specifically addressed elsewhere in this paper. Unfortunately, the notation and terminology are not consistent between these two references. However, we are interested in the observation that the phenomenon of Theorem 6 always occurs in these families and, consequently, in inverse limits using single members of such families, B-J-K continua occur and signal the onset of indecomposability either of the core or of the inverse limit itself. Consequently, we will not attempt to resolve the differences here. Instead, we identify the salient features of full (transition) families necessary to argue Theorem 9 and leave matters there. Suppose $\left\{f_{\lambda} \mid \lambda_{0} \leq \lambda \leq \lambda_{1}\right\}$ is a full (transition) family of mappings such that $f_{\lambda}$ maps the interval $\left[a_{\lambda}, b_{\lambda}\right]$ onto itself. Both sources require the members of the family to be unimodal and have negative Schwarzian derivative (the Schwarzian derivative of $f$ at $x$ is $\left.\frac{f^{\prime \prime \prime}(x)}{f^{\prime}(x)}-\frac{3}{2}\left(\frac{f^{\prime \prime}(x)}{f^{\prime}(x)}\right)^{2}\right)$. As above, we denote the single critical point between $a_{\lambda}$ and $b_{\lambda}$ by $c_{\lambda}$ (with the subscript $\lambda$ included on the critical point even though in the two sources this point remains constant, 0 in [3] and $\frac{1}{2}$ in [5]). In Theorem 9, we shall restrict our attention to full (transition) families of Type (1) or of Type (2). In case of Type (1) or Type (2) unimodal maps on an interval, denote the first point of $\left(c_{\lambda}, b_{\lambda}\right)$ of period 2 by $q_{\lambda}$ and note that in the families 
identified here and in Section 6, this is actually a fixed point for $f_{\lambda}$. When reading [3], the maps are Type (1) on [1-a,1], while in [5], they are Type (2) on $[0, \lambda]$ but of Type $(1)$ on $\left[f_{\lambda}(\lambda), \lambda\right]$. According to Collet and Eckmann [3, Proposition III.1.2, p. 174], the kneading sequences $R L R C$ and $R L^{\infty}$ both occur in the family. (The kneading sequence is a record of the relation of the orbit of $c$ to $c$ with an $R$ meaning that the term of the orbit is greater than $c$, an $L$ meaning that the term of the orbit is less than $c$, and a $C$ meaning the term of the orbit is $c$. E.g., the kneading sequence $R L R C$ for $f$ means that $f(c)>c, f^{2}(c)<c, f^{3}(c)>c$, and $f^{4}(c)=c$.) Devaney [5, p.149], on the other hand, makes it clear that one needs continuity in the parameter, $\lambda$. Using these features of full (transition) families, we prove the following:

Theorem 9. If $\left\{f_{\lambda} \mid \lambda_{0} \leq \lambda \leq \lambda_{1}\right\}$ is a full (transition) family of mappings, then there exists a parameter value, $\lambda$, such that $f_{\lambda}^{2}\left(b_{\lambda}\right)=q_{\lambda}$.

Proof. We shall restrict our attention to full (transition) families where all members of the family are of the same type and this is Type (1) or Type (2). Consequently, on $\left[c_{\lambda}, b_{\lambda}\right]$ the mapping $f_{\lambda}$ is non-increasing. Observe that, since there is a parameter value $\mu$ where the kneading sequence $R L R C$ occurs, $f_{\mu}^{2}\left(b_{\mu}\right)>c_{\mu}$. If $f_{\mu}^{2}\left(b_{\mu}\right) \leq q_{\mu}$, then, since $q_{\mu} \leq p_{\mu}, f_{\mu}^{3}\left(b_{\mu}\right)>p_{\mu}$. However, this involves a contradiction since the fourth term of the kneading sequence is a $C$. Thus, $f_{\mu}^{2}\left(b_{\mu}\right)>q_{\mu}$. At the parameter value $\lambda_{1}, R L^{\infty}$ is the kneading sequence. Here, $f_{\lambda_{1}}^{2}\left(b_{\lambda_{1}}\right)<q_{\lambda_{1}}$. Thus, by the continuity in the parameter $\lambda$, there is a parameter value $\lambda$ where $f_{\lambda}^{2}\left(b_{\lambda}\right)=q_{\lambda}$.

Remark. As a consequence of this theorem we see that in every full family of mappings there is a parameter value for which the inverse limit contains a union of two B-J-K continua intersecting at a point and beyond which the mapping has a periodic point of odd period greater than one.

\section{SPECIFIC FAMILIES}

In this section we discuss some uses of the theorems of this paper in four specific families of mappings. The investigation of inverse limits in these families spawned this paper. The four families are:

the logistic family on $[0,1]$ given by

$$
f_{\lambda}(x)=4 \lambda x(1-x), \quad 0 \leq \lambda \leq 1 ;
$$

the tent family on $[0,1]$ given by

$$
T_{\lambda}(x)=\left\{\begin{array}{ll}
2 \lambda x, & \text { if } 0 \leq x \leq \frac{1}{2} \\
2 \lambda(1-x), & \frac{1}{2} \leq x \leq 1,
\end{array} \text { for } \frac{1}{2} \leq \lambda \leq 1 ;\right.
$$

the family $\mathscr{F}$ on $[0,1]$ given by

$$
f_{t}(x)=\left\{\begin{array}{ll}
2 x, & \text { if } 0 \leq x \leq \frac{1}{2} \\
2(t-1)(x-1)+t, & \frac{1}{2} \leq x \leq 1,
\end{array} \text { for } 0 \leq t \leq 1 ;\right.
$$

and the family $\mathscr{G}$ on $[0,1]$ given by

$$
g_{t}(x)=\left\{\begin{array}{ll}
2(1-t) x+t, & 0 \leq x \leq \frac{1}{2} \\
2(1-x), & \frac{1}{2} \leq x \leq 1,
\end{array} \text { on }[0,1] \text { for } 0 \leq t \leq 1\right.
$$


The members of the logistic family are Type (2) unimodal mappings on $[0, \lambda]$ for all $\lambda$ as are the members of the tent family. The members of $\mathscr{F}$ are Type (2) and the members of $\mathscr{G}$ are Type (1) for all $t$.

We begin with the logistic family. The non-zero fixed point for the mapping $f_{\lambda}$ in the logistic family is $p=1-\frac{1}{4 \lambda}$. The only parameter value for which $f_{\lambda}^{2}(\lambda)$ is a true period two point is $\lambda=\frac{1+\sqrt{5}}{4}$. (Note. At this parameter value the period 2 point is $q=\frac{1}{2}$ and the inverse limit is a ( $\left.\sin \frac{1}{x}\right)$-curve). The conditions in the hypothesis of Theorem 4 are met only at the parameter value which is the solution $\lambda_{M_{1}}$ to $f_{\lambda}^{2}(\lambda)=1-\frac{1}{4 \lambda}$. Solving for $\lambda_{M_{1}}$ amounts to solving the cubic polynomial $8 \lambda^{3}-4 \lambda^{2}-2 \lambda-1=0$. The solution is $\frac{1}{6}+\frac{2}{3 \sqrt[3]{19+\sqrt{297}}}+\frac{\sqrt[3]{19+\sqrt{297}}}{6}$, which is approximately 0.91964 . Thus, by Theorem 6 , members of the logisitic family have periodic points of odd period only for parameter values greater than $\lambda_{M_{1}}$.

For the member $T_{\lambda}$ of the tent family, the non-zero fixed point is $p=\frac{2 \lambda}{2 \lambda+1}$. There is no true period two point in $\left[\frac{1}{2}, p\right]$ for any parameter value. The conditions in the hypothesis of Theorem 4 are met only at the parameter value $\lambda=\sqrt{2}$. Thus, by Theorem 6 , members of the tent family have periodic points of odd period only for parameter values greater than $\sqrt{2}$.

For the the member $f_{t}$ of the family $\mathscr{F}$, the non-zero fixed point is $p=$ $\frac{t-2}{2 t-3}$. The only true period two points occur for $t=\frac{1}{2}$ at which every point of $\left[\frac{1}{2}, p\right)$ is a period two point. (Note. At this parameter value the inverse limit is, once again, homeormorphic to a $\left(\sin \frac{1}{x}\right)$-curve). The conditions in the hypothesis of Theorem 4 are met only at the parameter value for which $f^{2}(1)=p$. This value is $t=\frac{7-\sqrt{17}}{8}$. Thus, by Theorem 6 , members of $\mathscr{F}$ have periodic points of odd period only for parameter values less than $\frac{7-\sqrt{17}}{8}$.

For the member $g_{t}$ of the family $\mathscr{G}$, the fixed point is $p=\frac{2}{3}$. There are no true period two points in $\left[\frac{1}{2}, \frac{2}{3}\right]$ for any member of $\mathscr{G}$. The conditions in the hypothesis of Theorem 2 are met only when $g_{t}(0)=\frac{2}{3}$. The solution is $t=\frac{2}{3}$. Thus, by Theorem 6 , the members of $\mathscr{G}$ have periodic point of odd period only for parameter values less than $\frac{2}{3}$.

\section{ACKNOWLEDGMENT}

The author acknowledges with thanks a conversation with Marcy Barge which improved this paper. The author also appreciates the conscientious job of the referee which also improved this paper.

\section{REFERENCES}

1. Marcy Barge and Joe Martin, Chaos, periodicity, and snakelike continua, Trans. Amer. Math. Soc. 289 (1985), 355-365.

2. Ralph Bennett, On Inverse Limit Sequences, Master's Thesis, University of Tennessee, 1962.

3. Pierre Collet and Jean-Pierre Eckmann, Iterated Maps on the Interval as Dynamical Systems, Birkhaüser, Basel, 1980.

4. James F. Davis, Confluent mappings on [0, 1] and inverse limits, Topology Proc. 15 (1990), $1-9$.

5. Robert L. Devaney, An Introduction to Chaotic Dynamical Systems, Benjamin, Menlo Park, 1986. 
6. W. T. Ingram, Concerning periodic points in mappings of continua, Proc. Amer. Math. Soc. 104 (1988), 643-649.

7. W. T. Ingram, Periodic points for homeomorphisms of hereditarily decomposable chainable continua, Proc. Amer. Math. Soc. 107 (1989), 549-553.

8. K. Kuratowski, Topology, Vol. 2, Academic Press, 1968.

9. D. P. Kuykendall, Irreducibility and indecomposability of inverse limits, Fund. Math 80 (1973), 265-270.

10. John Milnor and William Thurston, On iterated maps of the interval, Lecture Notes in Math., vol. 1342, Springer-Verlag, Berlin and New York, 1986, pp. 465-563.

Department of Mathematics and Statistics, University of Missouri-Rolla, Rolla, MissOURI 65401

E-mail address: INGRAMOUMR. EDU 